\title{
Is Surgical Intervention Safe and Effective in the Treatment of Myasthenic Blepharoptosis? A Multicenter Survey in Japan
}

\author{
Yusuke Shimizu $^{\text {a }}$ Shigeaki Suzuki $^{\text {b }}$ Kimiaki Utsugisawac ${ }^{c}$ Tomihiro Imai $^{d}$ \\ Hiroyuki Murai $^{\mathrm{e}}$ Yuriko Nagane $^{c}$ Emiko Tsuda $^{d}$ Tomohisa Nagasao $^{a}$ Hisao Ogata $^{a}$ \\ Masaki Yazawa ${ }^{a}$ Norihiro Suzuki ${ }^{b}$ Kazuo Kishia \\ Departments of a Plastic and Reconstructive Surgery and b Neurology, Keio University School of Medicine, Tokyo, \\ 'Department of Neurology, Hanamaki General Hospital, Hanamaki, dDepartment of Neurology, Sapporo Medical \\ University, Sapporo, and e Department of Neurology, lizuka Hospital, lizuka, Japan
}

Dear Sir,

Myasthenia gravis (MG) is an antibodymediated, neuromuscular transmission disorder. Its clinical manifestations range from ocular myasthenia, which can be visually disabling, to myasthenic crisis, with patients experiencing life-threatening respiratory insufficiencies [1]. Several effective medical treatments are available for the ocular symptoms of MG [2-6]; however, physicians often struggle to treat patients with longstanding blepharoptosis [7-9]. Moreover, although blepharoptosis surgery is occasionally applied in such cases $[10,11]$, the indications and clinical effectiveness of blepharoptosis surgery for MG patients remains unclear. In the present study, we retrospectively investigated the clinical features of blepharoptosis surgery by interviewing 19 Japanese patients with MG.

\section{Methods}

We examined 676 Japanese patients with MG (240 men, 436 women; mean age: 57.6 years) who were consecutively evaluated between April and July 2012 at the 11 neurological centers constituting the Japan MG Registry Study Group. All of the patients were interviewed about the clinical features of their MG and whether they had undergone blepharoptosis surgery. A total of 19 patients $(2.8 \%$; 7 men, 12 women; mean age: 65.7 years) had undergone blepharoptosis surgery, and were further evaluated by using a questionnaire.

Questions regarding the timing of the surgery (relative to MG onset), the ease of eyelid opening [over both the short term ( $\leq 1$ year) and long term ( $>1$ year), postoperatively], aesthetic outcome (scarring), overall satisfaction with the surgery, and any noted complications. Three-point Likert scales were used to assess the ease of eyelid opening (much easier, easier, worse), scarring (very minimal, minimal, visible), and overall satisfaction with the surgery (very satisfied, satisfied, dissatisfied). To evaluate scarring, the patients answered the questionnaires with their attending neurologists in the out-patient clinics. The actual surgeons were not present to help in obtaining unbiased opinions from the patients. All clinical information was collected after the patients had provided written informed consent, and the study was approved by the institutional review boards of each hospital.

\section{Results}

The clinical features of MG and the postoperative status of these 19 patients are summarized in table 1 . The time to surgery after the MG diagnosis ranged from 2 to 30 years. However, 5 patients (26\%) underwent blepharoptosis surgery before being diagnosed with MG. The degree of shortterm ( $\leq 1$ year) postoperative eyelid opening, compared with the preoperative status, was much easier in 12 patients $(63 \%)$ and easier in 7 patients (37\%). The ease of longterm ( $>1$ year) postoperative eyelid opening, compared with the preoperative status, was much easier in 7 patients (37\%), easier in 10 patients $(53 \%)$, and worse in 2 patients $(11 \%)$. In terms of aesthetic outcomes, postoperative scarring was very minimal in 15 patients (79\%), minimal in 2 patients $(11 \%)$, and visible in 2 patients (11\%). The overall satisfaction levels were 'very satisfied' in 11 patients (58\%), 'satis-

\section{KARGER}

E-Mail karger@karger.com

www.karger.com/ene (c) 2014 S. Karger AG, Basel

0014-3022/14/0716-0259\$39.50/0
Yusuke Shimizu, MD, $\mathrm{PhD}$

Department of Plastic and Reconstructive Surgery Keio University School of Medicine

35 Shinanomachi, Shinjuku-ku, Tokyo 160-8582 (Japan)

E-Mail yyssprs@gmail.com 
Table 1. Clinical features of the 19 MG patients who underwent blepharoptosis surgery

\begin{tabular}{ll}
\hline Clinical features & Values \\
\hline Age of onset, years & $\begin{array}{l}22-79 \text { (aver- } \\
\text { age: } 65.7)\end{array}$ \\
Gender (m/f), n & $7 / 12$ \\
MGFA, n & 8 \\
I & 5 \\
II & 4 \\
III & 1 \\
IV & 1 \\
V & \\
AChR, n & 13 \\
Positive & 6 \\
Negative & \\
Time to surgery, n & 5 \\
Before MG diagnosis & 5 \\
After MG diagnosis & \\
$\quad<2$ years & 0 \\
$\quad$ 2-5 years & 9 \\
$\quad$ 5 years & 5 \\
\hline
\end{tabular}

AchR = Acetylcholine receptor; MGFA = Myasthenia Gravis Foundation of America.

fied' in 4 patients (21\%), and 'dissatisfied' in 4 patients $(21 \%)$. Three of the 4 patients who answered 'dissatisfied' had undergone blepharoptosis surgery prior to a definitive MG diagnosis. Complications were not reported by any patient.

\section{Discussion}

Many patients with MG experience longstanding blepharoptosis, despite undergoing multiple medical procedures, and this condition substantially reduces the patient's quality of life. Although several reports have indicated that blepharoptosis surgery is suitable in such cases, its fre- quency, effectiveness, and overall satisfaction levels remain unclear.

In the present study, 19 (2.8\%) of the $676 \mathrm{MG}$ patients underwent blepharoptosis surgery, a rate that is much lower than the first author (a plastic surgeon) had expected. A possible explanation for this unexpectedly low rate is that only a few reports and reviews have recommended blepharoptosis surgery as a suitable treatment for myasthenic blepharoptosis [10, 11]. However, our study revealed that blepharoptosis surgery has a role as a safe and effective treatment for longstanding myasthenic blepharoptosis. All of the patients reported easier postoperative eyelid opening within the first year, and 17 of the 19 patients (89\%) reported easier eyelid opening over the long term. In addition, postoperative scarring was very minimal in the majority of patients (89\%). Moreover, most patients $(79 \%)$ were more than satisfied with their surgical outcomes.

Among the 4 patients $(21 \%)$ who were dissatisfied with their surgical outcomes, 3 had undergone blepharoptosis surgery before being definitively diagnosed with MG. If the 5 patients who underwent the surgery before diagnosis were excluded, 11 (79\%) among the 14 remaining patients were 'very satisfied', and 2 (14\%) were 'satisfied'. In terms of overall satisfaction, 14 (93\%) out of 15 patients were more than 'satisfied' with the outcome of the blepharoptosis surgery. The results hint that blepharoptosis surgery might be a good treatment choice for myasthenic blepharoptosis, although a limitation of our study is that only a small number of patients actually had the operation.

In the present study, major complications, such as lagophthalmos or exposure keratopathy, were not reported by any of the patients with MG. Although various minor complications are not entirely evident from the study, we believe that the postoperative complication rate might not be very different from those for patients without MG if a trained plastic surgeon performs the operation under close supervision of a neurologist and there has been an accurate diagnosis.

Through this multicenter study, we retrospectively found that the 19 operated patients were not selected for blepharoptosis surgery under explicit criteria. Hence, we propose here criteria for the surgical treatment of longstanding blepharoptosis based on our findings and institutional experience. We suggest that this procedure is indicated in cases where the MG patient is in a stable general condition, the MG patient has had at least 2 years of prior medical treatment by neurologists before surgery, the blepharoptosis is continuous and shows minimal circadian changes, the patient's quality of life is severely disrupted by the blepharoptosis, and the surgery is to be performed by a trained surgeon under proper supervision of a neurologist.

Of the $676 \mathrm{MG}$ patients retrospectively evaluated, 69 (10\%) had a history of MG greater than 2 years from onset and had persistent blepharoptosis (a quantitative MG score of 3 for the blepharoptosis). Hence, these patients might fulfill our criteria for a blepharoptosis operation, and there may be more patients who are candidates for surgical intervention. We recommend that neurologists and surgeons collaborate more closely and discuss the comprehensive treatment plan to increase the quality of life of MG patients.

\section{Acknowledgements}

This work was supported by a grant from the Japanese Ministry of Education, Science, Sports and Culture (No. 23591255) and a Neuroimmunological Disease Research Committee grant from the Japanese Ministry of Health, Labour and Welfare.

\section{Disclosure Statement}

All authors have no conflict of interest.
References
1 Kumar V, Kaminski HJ: Treatment of myasthenia gravis. Curr Neurol Neurosci Rep 2011;11:89-96.

$\checkmark 2$ Meriggioli MN, Sanders DB: Autoimmune myasthenia gravis: emerging clinical and biological heterogeneity. Lancet Neurol 2009;8: 475-490.
-3 Benatar M, Kaminski HJ, Quality Standards Subcommittee of the American Academy of Neurology: Evidence report: the medical treatment of ocular myasthenia (an evidencebased review): report of the Quality Standards Subcommittee of the American Academy of Neurology. Neurology 2007;68:2144-2149. 
4 Wolfe GI, Barohn RJ, Foster BM, Jackson CE, Kissel JT, Day JW, Thornton CA, Nations SP, Bryan WW, Amato AA, Freimer ML, Parry GJ, Myasthenia Gravis-IVIG Study Group: Randomized, controlled trial of intravenous immunoglobulin in myasthenia gravis. Muscle Nerve 2002;26:549-552.

5 Yagi Y, Sanjo N, Yokota T, Mizusawa H: Tacrolimus monotherapy: a promising option for ocular myasthenia gravis. Eur Neurol 2013;69:344-345.
6 Nagane Y, Suzuki S, Suzuki N, Utsugisawa K: Early aggressive treatment strategy against myasthenia gravis. Eur Neurol 2011;65:1622.

7 Kaminski HJ, Daroff RB: Treatment of ocular myasthenia: steroids only when compelled. Arch Neurol 2000;57:752-753.

8 Roach ES: Treating ocular myasthenia gravis with inadequate evidence. Arch Neurol 2007; 64:1794-1795.
9 Nagane Y, Utsugisawa K, Suzuki S, Masuda M, Shimizu Y, Utsumi H, Uchiyama S, Suzuki $\mathrm{N}$ : Topical naphazoline in the treatment of myasthenic blepharoptosis. Muscle Nerve 2011;44:41-44.

10 Evoli A, Batocchi AP, Minisci C, Di Schino C, Tonali P: Therapeutic options in ocular myasthenia gravis. Neuromuscul Disord 2011; 11:208-216.

11 Bradley EA, Bartley GB, Chapman KL, Waller RR: Surgical correction of blepharoptosis in patients with myasthenia gravis. Ophthal Plast Reconstr Surg 2001;17:103-110. 